\title{
Erratum to: Joint Hyponormality of Rational Toeplitz Pairs
}

\author{
In Sung Hwang and Woo Young Lee
}

\section{Erratum to: Integr. Equ. Oper. Theory (2009) 65:387-403 DOI 10.1007/s00020-009-1729-3}

In Theorem 1 of the article "Joint Hyponormality of Rational Toeplitz Pairs" [1], the condition ' $\phi$ and $\psi$ have a common pole' has to be assumed.

Due to a technical problem, we omitted an assumption in Theorem 1 of [1]. For the sake of completeness, we restate Theorem 1, in its correct form.

Theorem 1. Let $\phi$ and $\psi$ be rational functions in $L^{\infty}$ which have a common pole. If $\mathbf{T}=\left(T_{\phi}, T_{\psi}\right)$ is hyponormal then $\phi-\beta \psi \in H^{2}$ for some constant $\beta$.

The proof of [1, Theorem 1] is correct and complete under the additional assumption ' $\phi$ and $\psi$ have a common pole.'

In turn, Corollary 2 of [1] should also have an additional assumption ' $\theta_{0}$ and $\theta_{2}$ are not coprime,' which is equivalent to the condition ' $\phi$ and $\psi$ have a common pole.'

We would remark that Corollary 2 of [1] is still a generalization of the case of trigonometric polynomial symbols because if $\phi$ and $\psi$ are trigonometric (non-analytic) polynomials then they have a common pole at $z=0$.

\section{Reference}

[1] Hwang, I.S., Lee, W.Y.: Joint hyponormality of rational Toeplitz pairs. Integr. Equ. Oper. Theory 65, 387-403 (2009)

\footnotetext{
The online version of the original article can be found under doi:10.1007/s00020-009-1729-3.
} 
In Sung Hwang

Department of Mathematics

Sungkyunkwan University

Suwon 440-746

Korea

e-mail: ihwang@skku.edu

Woo Young Lee $(\bowtie)$

Department of Mathematics

Seoul National University

Seoul 151-742

Korea

e-mail: wylee@math.snu.ac.kr 